http://dx.doi.org/10.18778/2196-8403.2007.05

\author{
ANDREAS DEGEN
}

\title{
Patria und Peitsche. Weiblichkeitsentwürfe in der deutschen Wanda-Figur des 19. Jahrhunderts
}

\begin{abstract}
Tematem artykułu jest recepcja legendy o krakowskiej księżniczce Wandzie w literaturze niemieckojęzycznej XIX w. Autor koncentruje się na przedstawieniu Wandy w kategoriach specyfiki płci jako uosobienia wyższości oraz jej przemiany z bohaterskiej dziewicy w erotyczną despotkę. Przedmiotem analizy są teksty Wernera, Schillera, von Arnima, Hauffa, von Chézy'ego, Heysa, Sacher-Masocha, Maya, Fontana i Jürgensa oraz występująca w nich postać Wandy w różnych formach kobiecości: jako dziewica, towarzyszka życia oraz despotyczna i wodzowska pani.

Der Aufsatz beschäftigt sich mit der Rezeption der legendären Krakauer Fürstin Wanda in der deutschsprachigen Literatur des 19. Jahrhunderts. Dabei steht die geschlechtsspezifische Darstellung Wandas als Figur der Überlegenheit und deren Transformation von einer heroischen Jungfrau zu einer erotischen Despotin im Zentrum. In Entwürfen von Weiblichkeit als Jungfrau, Gefährtin und Herrin wird die Figur der Wanda in Texten von Werner, Schiller, v. Arnim, Hauff, v. Chézy, Heyse, Sacher-Masoch, May, Fontane und Jürgens untersucht.

The article deals with the adoption of the legendary Wanda, Princess of Krakow, in the German-speaking literature of the 19th century. It focuses on the gender-specific modelling of Wanda as a character of superiority and her transformation from a heroic virgin to an erotic despot. In terms of femininity, such as virgin, companion and mistress, the character of Wanda is discussed in texts by Werner, Schiller, v. Arnim, Hauff, v. Chézy, Heyse, Sacher-Masoch, May, Fontane and Jürgens.
\end{abstract}

\section{Herkunft und Merkmale der Figur}

Die mittelalterliche Legende von Wanda, Tochter und Nachfolgerin des Krakauer Gründungsfürsten Krak, die die Werbung eines deutschen Ritters ablehnt, ihn besiegt und sich anschließend durch einen Sprung in die Weichsel 
den Göttern opfert, gehört zu den bekanntesten politischen Mythen Polens. Bearbeitungen hat sie in der polnischen Literatur erwartungsgemäß in großer Zahl gefunden. ${ }^{1}$ Dass sie jedoch, trotz ihrer antideutschen Tendenz, auch innerhalb der deutschsprachigen Literatur rezipiert wurde, ist kaum bekannt. Von wenigen Untersuchungen einzelner deutscher Wanda-Texte abgesehen, ist die bis in die Gegenwart reichende eigenständige deutschsprachige Rezeption der polnischen Heroin unerforscht. ${ }^{2}$ Angelegt ist die Wanda-Figur als Figur der Überlegenheit: Als Polin siegt sie über den Deutschen, als Frau über den Mann, als pflichtbewusste Herrscherin über ihre Gefühle. Die semantischen Felder, in denen die Überlegenheit der Wanda-Figur Gestalt gewinnt, sind Kampf, Sittlichkeit und Erotik, wobei deren jeweiliger Einfluss auf die konkrete Figurenkonzeption von Text zu Text unterschiedlich akzentuiert sein kann. Die spezifische Überlegenheit Wandas wird bereits in den polnischen Bearbeitungen der Legende aus einer Verbindung traditionell weiblicher und traditionell männlicher Qualitäten hergeleitet. Sie verfügt über Sinnlichkeit wie über Vernunft, ihre Erscheinung ist von reizender Anmut, zugleich sind ihre Handlungen von irritierender Rationalität: „Vanda potens forma, Vanda potens animo" (CHOCHANOvius 1987:159), heißt es bereits in einer Elegie des polnischen Dichters Jan Kochanowski aus dem 16. Jahrhundert. Eine Verbindung weiblicher Schönheit einer- und politischer und militärischer Professionalität andererseits ist für die Darstellung einer Herrscherin etwa der Barockzeit (,femme forte') nicht ungewöhnlich (KoLLMANN 2004:36). Allerdings scheint im Falle der Kronprinzessin Wanda schon lange vor der Etablierung des Typus der ,femme fatale" in den europäischen Literaturen des 19. Jahrhunderts in einer solchen Verbindung ein - aus männlicher Sicht - unheilvolles Moment entwaffnender grausamer Überlegenheit zumindest angelegt zu sein. So heißt es beispielsweise in Samuel Friedrich Lauterbachs Pohlnischer Chronicke von 1727 über die polnische Wanda: „Sie soll eine ausbündig-schöne, und dabey hoch-verständige Princeßin, gewesen seyn, davon sie auch ihren Nahmen herhaben soll, der so viel heist, als hamus, ein Hacken, oder Angel, weil sie durch ihre ungemeine Freundlichkeit, aller Gemüther an sich gezogen." (LAUTERBACH 1993:263) Die reizende und zugleich verderbende Anziehungskraft,

1 Eine - fast ausschließlich polnische Texte berücksichtigende - Übersicht bis zum Jahr 1923 bietet MoRTKOWICZÓwnA (1927).

2 Zu nennen ist vor allem Gerard Koziełek, der seit seiner Arbeit über Werners Dramen wiederholt auf die deutsche Wanda-Rezeption um 1800 aufmerksam gemacht hat (KoZIEŁEK 1967:229-233; KoZIEŁEK 1976; KozIEŁEK 1989:29-31), außerdem KLIN (1996) und LIEDKE (1965). 
auf die dieser - nicht der einzige - Erklärungsversuch des Namens Wanda hindeutet, wird unter dem Einfluss des romantischen Weiblichkeitsstereotyps der dämonischen Frau im Verlauf der zweiten Hälfte des 19. Jahrhunderts zu einem prägenden Merkmal der Wanda-Figur.

Die Rezeption und Transformation der Wanda-Figur in der deutschsprachigen Literatur des 19. Jahrhunderts kann an unterschiedlichen Darstellungsvarianten der Figur verfolgt werden, die im Weiteren unter den Stichworten Jungfrau, Gefährtin und Herrin in chronologischer Folge beschrieben werden. Dieser Systematisierung ist das Verhältnis zwischen den beiden Protagonisten zugrunde gelegt: 1. Es kommt zu keiner Verständigung über die gegenseitigen Gefühle oder diese bzw. ein männlicher Protagonist werden nicht erwähnt (Jungfrau); 2. es kommt zu einer Verständigung über die gegenseitigen Gefühle, beide Figuren verkehren gleichrangig miteinander (Gefährtin); 3. es kommt zu einer Verständigung über die gegenseitigen Gefühle, die weibliche Protagonistin nimmt (freiwillig oder unfreiwillig) eine dominante, auch mit Gewalt gegen den Mann verbundene Position ein (Herrin). Die Zuordnung der einzelnen Texte ist allerdings nicht kategorisch aufzufassen, da es zwischen den verschiedenen Wanda-Figuren Berührungs- und Überschneidungsbereiche gibt, wie dies bei einer gemeinsamen Stoff- bzw. Figurentradition kaum anders $\mathrm{zu}$ erwarten ist. Nicht auf die Zuordnung kommt es letztendlich an, sondern auf die in der Gesamtheit erkennbaren chronologischen Transformationsprozesse in der Figurengestaltung. Diese Transformationsprozesse, die zu einer differenten Verteilung der als konstitutiv für die Figur angesehenen Merkmale Gewalt (kämpferische Überlegenheit), Affektkontrolle (sittliche Überlegenheit) und Anziehung (erotische Überlegenheit) führen, sind nicht als eigendynamischer Entwicklungs- und Modifizierungsprozess eines literarischen Motivs zu verstehen. Vielmehr steht die Figurenkonzeption der Wanda ähnlich wie bei Judith, Salome oder anderen weiblichen Figuren der Überlegenheit in einem beständigen Wechselverhältnis zu (dominanten oder marginalen) Weiblichkeitsdiskursen. Für das 19. Jahrhundert wäre vor allem auf das eine fundamentale Geschlechterdifferenz behauptende bürgerliche Ideal der Frau zu verweisen, das im Verlauf der ersten Hälfte des 19. Jahrhunderts zum gesellschaftlich einflussreichsten Weiblichkeitsentwurf wird. Weiblichkeit wird darin weitgehend auf passiv empfangende, hingebungsvoll liebende, dem privaten Bereich verhaftete und letztlich asexuell vorgestellte Gattinnen und Mütter beschränkt (BRUNS / PARR / WÜLFING 1991:131-134; SCHABERT 1995: 172f.): „In einer Epoche, die ein extrem gespaltenes Verhältnis zur Sexualität besaß, feierte man sie als Repräsentantinnen menschlicher Selbstzucht, als Heilsbringerinnen, die die sexuell ausschweifenden Männer versittlichen und 
zivilisieren sollten.“ (FREVERT 1995:220f.) So unterschiedlich die Konzeptionen der vorgestellten Wanda-Figur im Einzelnen auch sein mögen, lassen sich diese doch mehrheitlich als Agentinnen dieses bürgerlichen Frauenideals verstehen. Für die Untersuchung werden Texte von Friedrich Schiller (1804), Zacharias Werner (1807), Achim von Arnim (1810), Wilhelm Hauff (1827), Wilhelm von Chézy (1831), Paul Heyse (1851), Leopold von Sacher-Masoch (1870), Karl May (1865/75), Theodor Fontane (1837/38 bzw. 1890) und C. Jürgens (1902) sowie ein anonymer Roman (1804) herangezogen. Damit werden die wichtigsten, jedoch nicht alle deutschsprachigen Wanda-Texte des 19. Jahrhunderts berücksichtigt (DEGEN 2007).

\section{Wanda als Jungfrau}

Die mittelalterlichen und frühneuzeitlichen Chroniken und Historien, die den Krakauer Gründungsmythos von König Krak und seiner Tochter Wanda überliefern, schweigen sich über deren Gefühle aus. Vielmehr werden die Freiheit ihres Volkes, die Einhaltung eines Keuschheitsgelübdes oder ihr männlichautonomer Charakter mit wechselnden Akzentuierungen als Gründe für ihre Abweisung des werbenden deutschen Ritters genannt. An diese Konzeption einer keine Liebe empfindenden oder diese verschweigenden Wanda schließen einige deutschsprachige Texte aus der ersten Hälfte des 19. Jahrhunderts an (Schiller, v. Arnim, Fontane, Heyse). Obgleich sie, bis auf Fontanes Jugendgedichte, der heroischen Konzeption der Vorlage folgen, lassen sich bei Arnim und Heyse bereits deutliche Einschränkungen des heroischen Ideals bemerken.

Friedrich Schillers Wanda-Ballade, wahrscheinlich 1804 niedergeschrieben, ist über das Stadium eines Entwurfs nicht hinausgekommen. Der Entwurf lässt als dramatischen Kern die Situation unmittelbar vor Wandas Sprung in die Weichsel erkennen: Die opferbereite Wanda steht auf der Brücke und spricht zu ihrem Volk. Entstanden ist Schillers Skizze in Zusammenhang mit seinem slawischen Drama Demetrius, die Konzeption der Wanda-Figur könnte seiner drei Jahre früher beendeten romantischen Tragödie Jungfrau von Orleans folgen. In dieser sieht Johanna schließlich von der Liebe zu ihrem Feind ab und erfüllt ihre Sendung, indem sie sich als Opfer darbringt und als solches verklärt wird. In Schillers Balladenentwurf will Wanda ,nichts wißen [!] von Liebe“, ihre „hohe, stolze, jungfräuliche Gesinnung“ ist allein auf „Freiheit u[nd] Ruhm“ gerichtet (SCHILLER 1993:438). Schiller hat aus seiner Quelle, der Pohlnischen Chronicke Lauterbachs, auch die Deutung des Namens übernommen: „Vanda heißt die Angel, sie hieß so weil sie sehr bezaubernd war" (SCHILLER 1993:438). Gänzlich ohne Empfindungen kommt auch die Wanda-Figur der 
Geschichte der verlorenen Erbprinzessin Wenda aus, die Achim von Arnim in seinen Roman Armut, Reichtum, Schuld und Buße der Gräfin Dolores von 1810 eingefügt hat. Vorlage ist der 1705 in Den Haag anonym herausgekommene Roman Venda, Reine de Pologne. Histoire Galante, der einer verwitweten anhaltinischen Prinzessin, offenbar Marie Eleonore von Anhalt-Dessau, verwitwet von Radziwill, gewidmet ist. Arnim erzählt allerdings aus der grotesk verzerrenden Perspektive eines jedem sittlichen Ideal unzugänglichen schweizerischen Geschäftsmannes, wodurch der spektakuläre Sprung Wandas in die Weichsel nur mehr als lächerliche und verwerfliche Tat einer religiösen Schwärmerin erscheint. Die heroische Tragik des Geschehens wird, da die Jungfrau dank einer monströsen Apparatur wieder zum Leben zurückfindet, als pfäffisches Blendwerk karikiert, wobei im Gesamtzusammenhang des arabesk montierten Romans diese Karikierung aufgehoben wird. Auf die Vorgeschichte des Selbstopfers geht - im Unterschied zur französischen Vorlage Arnims Wanda-Version ebenso wenig ein wie auf die Figur des männlichen Werbers. Werbung und unerfüllte Liebe stehen in vier Wanda-Gedichten des achtzehnjährigen Theodor Fontane im Mittelpunkt. Die 1837/38 entstandenen, eine Usedomer Urlaubsbekanntschaft verarbeitenden Gedichte Der Kastanienbaum, Trauriges Erwachen, Trost und Auf dem See wenden sich, ohne einen weiteren Bezug zur Wanda-Legende, an eine Geliebte namens Vanda bzw. Wanda. Diese hat den Sprecher des Gedichtes grußlos verlassen und seine Gefühle abgewiesen, trauernd erinnert er sich an ihre „Unschuld“, „Reinheit“ und „Seelengüte“ (FONTANE 1964:596). Den seltenen Fall einer ausschließlich weiblichen Perspektivierung des Liebesverhältnisses bietet Paul Heyses vermutlich 1851 entstandene Wanda-Ballade. Im Unterschied zu den bisher behandelten Texten nimmt Heyse das in der deutschen Wanda-Literatur etablierte Motiv der wechselseitigen Liebe auf, mit der Einschränkung allerdings, dass Wanda sich in ihrer Gegenliebe dem Geliebten nicht zu erkennen gibt. Im Konflikt zwischen ihren Gefühlen und dem, was sie ihrem Volk und dessen heidnischen Göttern schuldig zu sein glaubt, sieht die Krakauer Fürstin im Tod den einzigen Ausweg. Ihr Tod hat jedoch nicht, wie beispielsweise in Zacharias Werners Wanda-Tragödie, den Charakter einer metaphysischen Hochzeit mit dem Geliebten. Auch feiert in ihm kein selbstloser Idealismus einen glänzenden Sieg wie bei Schiller. Der einsame Sprung von Heyses unheroischer Wanda wird stattdessen als nächtliche Verzweiflungstat dargestellt, mit der die ihren Gefühlen unterliegende Fürstin dem gänzlichen Verlust ihrer Selbstkontrolle zuvorzukommen versucht. Unter dem Blick Rüdigers droht ihre Stärke „wie ein Schnee im Mai'n“ (HeYse 1980:133) zu schmelzen. Heyse fasst den ganz ins Innere der Protagonistin verlagerten Konflikt zwischen 
Pflicht und Neigung in die Schwellenmetapher des Gittertores, durch das Wanda den die Stadt Krakau belagernden Feind erblickt und begehrt, das sie ihm aber nicht öffnen darf. Der Sprung in den Tod, der durch keine christliche Verklärung aufgehoben wird, geht dem Eingeständnis ihrer Liebe voraus. Mit dieser Verweigerung einer idealistischen Perspektive auf die in ein ,freies Heidengrab" (HEYSE 1980:133) sinkende Wanda in Heyses Ballade endet in der Mitte des 19. Jahrhunderts die deutschsprachige Rezeption der WandaFigur in ihrer traditionellen Form.

\section{Wanda als Gefährtin}

Die Überlegenheit der Wanda-Figur steht in der deutschsprachigen Rezeption oft in einem Spannungsverhältnis zu einer individuell angestrebten Partnerschaft mit dem männlichen Protagonisten. Ein derartiges partnerschaftliches Verhältnis ist entweder aus patriotischen Gründen verwehrt (anonymer Roman, Hauff), aufgrund seiner ökonomischen statt einer emotionalen Basis nur vorgetäuscht (Fontane), oder es wird unter männlicher Führung in Form einer (klein)bürgerlichen Ehe verwirklicht (May). In diesen Wanda-Texten erwidert Wanda die Liebe des Mannes. Ihre Überlegenheit wird durch die emotionale Annäherung abgeschwächt oder ist, wie ansatzweise bei May, vor allem aber bei Hauff und Fontane, zugunsten des männlichen Protagonisten aufgegeben. Der 1804 anonym in Wien erschienene zweiteilige Roman Wanda, Fürstin von Krakau, oder die Opfer der Liebe. Nach der wahren Geschichte romantisch bearbeitet folgt, von der wechselseitigen Liebe abgesehen, in seinen Grundzügen der traditionellen Wanda-Legende. Wanda wird als ausgesprochen selbstbewusste, auf ihrer dynastischen und persönlichen Autonomie bestehende Thronfolgerin eingeführt. Sie verfügt über große Schönheit und Milde wie über einen „männlichen Geist“ (ANONYMUS 1804:75), kann fechten wie ein Mann und reitet zur Jagd aus. Allerdings weiß sie zum Bedauern der polnischen Brautwerber nicht, „was Liebe sey“ (ANONYMUs 1804:76), vielmehr ist ihr die „Verbindung mit einem Manne nichts als eine unerträgliche Knechtschaft" (ANONYMus 1804:8). Damit wird sie für die zahlreichen in- und ausländischen Thronaspiranten ein Hindernis auf dem Weg zur Macht. Erst als Wanda während einer Jagd durch den ihr bislang unbekannten Ritter Rithogar gerettet wird, entflammt ihr Herz. Da sie aber, um ihre Heiratsunwilligkeit innenpolitisch zu legitimieren, einst einen Keuschheitsschwur geleistet hat, besteht sie nach dem ersten Kuss darauf, sich wieder von dem Geliebten zu trennen. Spätere Annäherungsversuche des ihr Falschheit unterstellenden Ritters wehrt sie ab. Als sie ihn auf eine Zweisamkeit im Jenseits vertröstet, nennt 
er sie eine „Schwärmerin“ bzw. eine „Grausame“ (ANONYMUS 1804:103, 105). In der vielsträngigen weiteren Romanhandlung sehen sich die getrennten Liebenden jeweils in ähnlicher Weise den Ränken ihrer Vasallen ausgesetzt, die aus eigenen Interessen auf eine nationale Heirat Wandas bzw. Rithogars drängen und dafür ihrerseits deutsch-polnische Allianzen zu schmieden beginnen. Als Wanda und Rithogar sich nach Jahren der Trennung inmitten einer Schlacht ihrer Völker unvermutet und vor Glück wie gebannt gegenüberstehen, wird Rithogar von einem deutschen Rivalen ermordet, Wanda bleibt wie betäubt vor Schmerz zurück. Wie zuvor in der Liebe wird nun in der Trauer Wandas außerordentliche Selbstkontrolle hervorgehoben, die ihre wahren Gefühle nicht nach außen dringen lässt. Nach dem Sieg der Polen und der Erfüllung ihrer herrschaftlichen Obliegenheiten springt sie, den Namen des Geliebten auf den Lippen, heiter in den Tod, dem sie als Vereinigung mit dem Geliebten entgegensieht. Diese anfangs auf Autonomie bestehende Wanda - sie bietet den drängenden Vasallen sogar ihren Verzicht auf die Herrschaft an wird zu einer disziplinierten Liebenden, die dem weniger beherrschten Geliebten trotz der räumlichen Distanz emotional näher zu sein scheint als dem eigenen Volk. Gleichfalls diszipliniert, ihrer Autonomie allerdings gänzlich benommen, ist die Wanda-Figur in der Binnenerzählung von Wilhelm Hauffs 1827 entstandener Novelle Die letzten Ritter von Marienburg. Entsagung von der Liebe und heroischer Tod zeichnen hier den männlichen Protagonisten aus, während Wanda als passive Dulderin seiner $\mathrm{Zu}$ - wie seiner Abwendung ganz an den Rand des Geschehens rückt. Eingebettet in eine Satire auf die Literaturvereine seiner Zeit lässt Hauff in der Novelle aus einem fiktiven historischen Roman vorlesen, dessen Handlung vom Ende des Deutschen Ritterordens und der Zerstörung der Marienburg 1457 erzählt. Männlicher Held dieses Romans ist Kuno von Elrichshausen. Er ist der Neffe des Hochmeisters, unterhält aber entgegen der Ordensregel ein heimliches Verhältnis mit Wanda, der Tochter des verräterischen, die Burg den Polen ausliefernden Kastellans. Mit der zeitlichen und räumlichen Adaption der Krakauer Gründungslegende kommt es auch zu einer Vertauschung der Figurenkonzeption: Subjekt der Handlung ist Kuno, der „voll schönen Glaubens an alles Edle und Reine“ (HAUFF 1970:606) zwischen seiner Liebe zu Wanda, seiner Freundschaft zu seinem Waffenbruder und seinen Pflichten gegenüber seinem Onkel bzw. den Ordensidealen hin und her gerissen ist. In den zusammengefasst vorgetragenen Passagen dieser Erzählung in der Erzählung erfährt die aufsteigende Wertehierarchie von Gemeinwohl, Freundschaft und Liebe eine markante Umbesetzung: Zunächst hat Kuno wegen seiner Liebe zu Wanda die Gemeinschaftswerte des Ordens missachtet. Als er von seinem im Kampf gegen die heidnischen Polen fallenden 
Waffenbruder erfährt, dass dieser gleichfalls, allerdings ohne ihnen nachzugeben, Neigungen zu Wanda empfunden habe, entsagt er in einem ,romantischen Entschluß“ (HAUFF 1970:625) auf immer der Liebe. Als die nun völlig verwaist dahinsiechende Wanda ihn bittet, sie vor dem endgültigen Rückzug des Ordens noch einmal aufzusuchen, willigt er zwar ein, verlässt sie aber nach wenigen Augenblicken, um bei der Verteidigung der Ordensfahne - Symbol der religiösen und sozialen Werte des Ordens - sein Leben zu lassen. Über Wanda, die lediglich als Objekt männlicher Entscheidungen fungiert, ist nichts weiter zu erfahren. Als Verkörperung eines unheroischen Fehltritts des Helden bleibt sie von den persönlichen (Freundschaft) und kollektiven (Orden) Männerbünden und deren Verdiensten ausgeschlossen. In ihrer Passivität und Zuordnung auf den privaten Bereich folgt die Figur dem bürgerlichen Frauenbild des 19. Jahrhunderts. Eine ganz andere, letztlich aber ebenfalls unterordnende Konzeption der Frauenfigur lässt Karl Mays Novelle Wanda erkennen. May veröffentlichte die Novelle zuerst im Jahr 1875, das erste, für die Figurenkonzeption entscheidende Kapitel entstand aber ungefähr zehn Jahre früher, wahrscheinlich im Nachklang des polnischen Aufstandes von 1863/64. Handlungsort der Detektivgeschichte ist eine Kleinstadt im sächsischen Erzgebirge. In dieser hält sich Wanda von Chlowicki, allgemein als die ,wilde Polin“ (MAY 1901:5) bezeichnet, mit ihrer Stiefmutter auf, um der Verlobung mit dem durch ihren verstorbenen Vater bestimmten Bräutigam Baron von Säumen entgegenzusehen. Zunächst scheint die Figur dem männlichen Protagonisten Emil Winter, eine für May charakteristische omnipotente Ich-Figur des Autors, in jeder Hinsicht ebenbürtig, mit ihm zeichnet sie sich vor allen anderen Figuren aus. Im Fortgang der abenteuerlichen Handlung rückt sie allerdings immer mehr in die Position einer bloßen Gehilfin des Mannes. Statt eines nationalen steht bei May ein sozialer Konflikt im Hintergrund des Geschehens, allgemein lässt sich die Überwindung vertikaler Distanz als eine wiederkehrende soziale Symbolstruktur des Textes auffassen. Während einer Vereinsfeier betritt der rußgeschwärzte, von einem Rettungseinsatz kommende Schornsteinfeger Winter den Festsaal. Er zeigt sich wenig später als unerwarteter und anfangs völlig aussichtslos erscheinender Mitbewerber um die Gunst der stadtbekannten Polin. Furchtlos und klug, edel und charmant, erwirbt sich Winter bald die Sympathien Wandas, rettet sie aus lebensgefährlichen Situationen und entlarvt den vermeintlichen Baron und Bräutigam als üblen Gauner. Nebenbei erkennt er in Wandas Stiefmutter eine Tante und in Wanda das ,wilde [...], reizende [...] Mädchen“ (MAY 1901:49) wieder, dem er in glücklichen Kinderjahren einmal im Wald begegnet war. Damit überwiegen auch hinsichtlich der sozialen Herkunft die Gemeinsamkeiten, so dass einem glücklichen Ende nichts 
mehr im Wege steht. Mays Novelle lässt sich als eine realistische, in die Gegenwart versetzte Variante von Werners sechzig Jahre früher entstandener Tragödie Wanda oder die Königin der Sarmaten lesen: Hier wie dort wird das gegenwärtig Trennende durch die Bewusstwerdung einer ursprünglichen Verbundenheit überwunden, wobei May eine innerweltliche Lösung, nämlich die Aufdeckung des falschen Scheins adliger Abstammung durch den Verdienstadel seines Unterschicht-Helden, bevorzugt. Gegenüber dem an allen Fronten siegreichen Winter weist die Figur der Wanda, die früheste Frauengestalt im Werk Mays, Inkonsistenzen auf. In den Text eingeführt wird sie als sittlich, körperlich, geistig und musisch hervorragende, weil wie ein Mann ausgebildete Frau. Zu ihrer anmutigen Schönheit, Beherztheit und inneren Noblesse tritt, ganz in der Tradition der Wanda-Legende, eine „scheinbar unweibliche [...] Vielseitigkeit und Selbständigkeit“ (MAY 1901:6). Die mit ihrer Ankunft im Städtchen ausgebrochene „Wandamanie“ (MAY 1901:5) ignoriert sie. Da sie jedoch Menschen „nach dem äußeren Scheine“ (MAY 1901:22) zu beurteilen pflegt, begegnet sie dem rußgeschwärzten Handwerker Winter zuerst mit ,jene[r] strenge[n] Kälte, hinter deren Schild die gekränkte Weiblichkeit sich so gern und erfolgreich flüchtet" (MAY 1901:20f.). May gibt, im Sinne seiner Unterschichtperspektive, die figurencharakteristische Zurückweisung durch die Frau als genderspezifische Eitelkeit einer Adligen aus, die erst durch den Großmut des männlichen Helden gebrochen wird. Verglichen mit den anfangs angeführten Qualitäten, bleibt die weibliche Figur innerhalb der gesamten Handlung allerdings erstaunlich passiv. Sie tritt lediglich als Objekt und Preis männlicher Unternehmungen in Erscheinung: Ihr Vater verfügt über sie, um eine Schuld zu tilgen, dem falschen Baron soll sie als Mittel zu einer Erbschleicherei dienen. Für Winter ist sie wiederholt Anlass, seine überragenden Fähigkeiten öffentlich unter Beweis zu stellen, bis sie ihm als Lohn für seine Heldentaten zufällt. Je deutlicher May seine Botschaft von der verkannten Größe darstellt, desto mehr verliert Wanda an Stärke und Autonomie. Die Figur der wilden Polin schrumpft auf die Rolle einer schutzbedürftigen Verwandten und fügsamen Erfüllungsgehilfin eines Provinzheroen und wird am Ende der kleinbürgerlichen Existenz an der Seite eines vielseitig talentierten Schornsteinfegers zugeführt. Noch drastischer ist die Diskrepanz zwischen der traditionellen Überlegenheit der Wanda-Figur und ihrer realen Abhängigkeit in Theodor Fontanes Berlin-Roman Stine dargestellt, entstanden zwischen 1881 und 1890. Wanda ist im Roman nur Nebenfigur. Die für beide Hauptfiguren tragisch durchgeführte Thematik standesübergreifender Liebe nimmt bei ihr einen komischen Charakter an. Wie bei May steht ein sozialer Konflikt im Hintergrund, allerdings bilden bei Fontane die männliche und die weibliche 
Position Gegensätze. Zwei der Unterschicht angehörende Schwestern und deren Freundin Wanda Grützmacher stehen in unterschiedlich gearteten Beziehungen zu zwei bejahrten Adligen und dem Neffen eines von diesen. Wanda, eine korpulente brünette Schönheit, ist Schauspielerin in einem Vorstadtheater. Ihr Auskommen bestreitet sie weniger durch drittklassige Auftritte als durch mehrere Gönner der gehobenen Gesellschaft. Die von ihr pragmatisch angegangenen Libertinagen würzt sie mit ,ganz unmotivierten Anstands- und Tugendrückfällen“ (FONTANE 2000:37), mit denen Fontane ironisch auf die traditionelle Wanda-Figur anspielt. Dies gilt noch mehr für Bemerkungen über Wandas recht elastische „Stellung zum ,Ideal““ (Fontane 2000:17) oder ihre Angewohnheit, sich mit dem Ausruf ,,Dafür laß ich mein Leben.““ von vermögenden Witwern die teuersten Speisen spendieren zu lassen. Anders als in den übrigen Wanda-Texten ist sie nicht als Polin oder Slawin ausgewiesen, doch stellt das von ihr vorgetragene Lied ,,Fordre niemand, mein Schicksal zu hören“" (FONTANE 2000:37) aus Karl von Holteis Singspiel Der alte Feldherr einen Bezug zur polnischen Freiheitsbewegung her. Ebenfalls auf das Vorbild der patriotischen Heroin zielt das während einer Plauderei der ungleichen Damen und Herren in einem mit gipsernen Polenfiguren bestückten Wohnzimmer aufgeführte Puppenspiel von Judith und Holofernes. Die dem Wanda-Stoff vergleichbare Legende über die Verführung und Enthauptung des assyrischen Feldherrn, mit der Judith ihr Volk rettete, wird von Wanda mit Puppen aus aufgespießten Kartoffeln aufgeführt. Die erzählerische Darstellung der Aufführung überblendet Wandas Gesicht mit der rotlippigen Puppe der Judith, wodurch das komische Missverhältnis zwischen der koketten Gelegenheitsprostituierten Wanda und dem idealistischen Heroismus ihrer Namensgeberin bzw. deren Parallelfigur Judith unterstrichen wird. Auch wenn Wanda Grützmacher nach ihrer Aufführung keck bemerkt, sie habe den Autor des Stücks selbstverständlich ,in der Gewalt“ (FONTANE 2000:35), haben ihre Männerbeziehungen nichts vom Heroismus noch von der Überlegenheit der Legendenfigur. Obgleich sie sich von älteren Gönnern hofieren lässt, ist sie in ökonomischer Hinsicht vollständig von diesen abhängig. Fontanes Wanda erweist sich somit als eine Möchtegern-Wanda, die ihre frivolen Anstandsappelle und erotischen Überlegenheitsposen zum Amüsement der sie aushaltenden Herren aufführt. Der Roman lässt keinen Zweifel an der Funktionsweise der patriarchalisch geprägten Hierarchie des deutschen Kaiserreiches, in der Wanda nur aufgrund ihrer emotionalen Verstellungskünste zu überleben vermag, während die beiden leidenschaftlich liebenden Hauptpersonen an den Standesgrenzen zugrunde gehen. Im Unterschied zu May ist Fontanes Wanda dem Mann nicht nur nicht überlegen, sondern unterlegen und gerade in den Freiheiten, die sie 
Patria und Peitsche. Weiblichkeitsentwürfe in der deutschen Wanda-Figur

sich herauszunehmen erlaubt, von seinen Vorgaben und Wünschen determiniert.

\section{Wanda als Herrin}

Die aufgezeigte Einfügung der Wanda-Figur in die heroischen oder erotischen Inszenierungen männlicher Akteure vollzieht sich in der zweiten Hälfte des 19. Jahrhunderts. Dieser Autonomieverlust lässt sich noch deutlicher an jenen Wanda-Figuren nachweisen, die als mütterliche Erzieherin oder grausame Herrin eine dominante bzw. vorgeblich dominante Position einnehmen. Als Figuren sittlicher Überlegenheit über den Mann folgen sie bei Werner und Chézy dem bürgerlichen Geschlechterdiskurs des frühen 19. Jahrhunderts, der die Frau als sittlich höchstes, den moralischen statt den natürlichen Trieben folgendes Wesen versteht (LANGE 1992:422), während sie in der erotischen Transformation bei Sacher-Masoch und Jürgens nicht nur die sittliche gegen eine erotische, sondern auch die überlegene gegen eine in Wahrheit subalterne Position eingetauscht haben. Das in der deutschsprachigen Rezeption des Wanda-Mythos prominenteste Werk ist Zacharias Werners romantische Tragödie Wanda oder die Königin der Sarmaten aus dem Jahr 1807. Erst sie machte, in der Variante einer tragischen Liebesgeschichte, den historischen Stoff in Deutschland größeren Kreisen bekannt. Bei Werner ist das Motiv für Wandas Heiratsunlust weder eine Unkenntnis der Liebe noch ein Verlangen nach persönlicher oder politischer Autonomie. Vielmehr versucht sie mit ihrem Keuschheitsgelübde, einem vor Jahren am Hof der Prager Königin Libussa geschlossenen ideellen Liebesbund mit einem unbekannten und mittlerweile für tot gehaltenen Ritter die Treue zu halten. Die metaphysische Tendenz ihrer Liebe bleibt auch dann erhalten, als sie in Rüdiger den geliebten Unbekannten erkennt. Sie muss, da sie ihre Heiratsunlust nur mit dem Versprechen, künftig allein ihr Volk zu lieben, gegenüber den sarmatischen Magnaten durchsetzen konnte, seine Werbung mit dem Verweis auf den ideellen Bund ablehnen. Erkenntnis und Erfüllung dieses über allen irdischen Bindungen stehenden Bundes bilden entsprechend Werners Zweihälftenlehre (KOZIEŁEK 1967:240) das programmatische Zentrum des Dramas. Dadurch wird die vordergründige Tragik in eine erlösende Wiederherstellung vorherbestimmter Ordnung umgedeutet. Das in diesem Sinne als Führerin und Vermittlerin des Mannes fungierende Weibliche wird in drei Figuren aufgespalten: in die als Geisterstimme erscheinende Königin Libussa, in die von ihr inspirierte Liebespriesterin Wanda und in die das erste Wiedersehen arrangierende Gärtnerin Ludmilla. Auf unterschiedlichen Ebenen werden alle drei weiblichen Figuren 
zu Agentinnen von Werners Liebesreligion, die den stolzen Krieger auf den Weg des demütigen Selbstopfers führen. Dieser hat seinerseits mit dem Eid, Wanda entweder zu erobern oder im Kampf sein Leben zu lassen, Vasallen für einen Heerzug nach Krakau geworben. Als Wanda und Rüdiger sich als Liebende wiedererkennen, sind also beide durch ein Versprechen an ihr Volk gebunden. Durch den losbrechenden Kampf ihrer Heere getrennt, stoßen sie wenig später erneut zusammen und schlagen, von der Unerfüllbarkeit ihrer Liebe rasend geworden, wild aufeinander ein. Nur die Intervention von Libussas Geist, der den ideellen Liebesbund als Erfüllung einer vorherbestimmten Vereinigung offenbart, führt zu einer Verständigung: „Alles, was erschaffen ward, / Ist von Ewigkeit gepaart“ (WERNER 1840/41:246). Die anschließende Verlobung nimmt den Charakter einer spirituellen Initiation Rüdigers an: Aus dem männlichen Bereich des Physischen und Trennenden wird er in den weiblichen Bereich des Metaphysisch-Verbindenden gehoben. Schon zuvor wird die Überlegenheit Wandas, die sich aus ihrem Wissen um eine höhere Ordnung speist, als spezifisch weibliche dargestellt. Als nämlich die sarmatischen Magnaten angesichts der Belagerung durch Rüdiger an der Schlagkraft ihrer gattenlosen Fürstin Zweifel anmelden (,Männer bändigt nur ein Mann!“ WERNER 1840/41:205), tritt Wanda ihnen furchtlos entgegen: „Dem Manne trotzt des Weibes stille Macht“ (WERNER 1840/41:213). Nur einmal gerät sie ins Wanken: Als Wanda dem geläuterten Geliebten den Todesstoß geben soll, mit dem er sich von seinen irdischen Bindungen lösen will, schreckt sie vor dem „Gräßlich-Schöne[n]“ (WERNER 1840/41:253) der Tat zurück und beruft sich auf die Schwachheit des Weibes. Jetzt ist es Rüdiger, der an das Heroische in ihr appelliert, so dass sie ihn als „Heldenweib“ tötet (WERNER 1840/41: 255). Sie folgt ihm wenige Tage später nach, von ihrem Volk verehrt und verklärt. Ihr Sprung durch die Flammen in die Weichsel wird als himmlische Hochzeit der „Götterbraut“ (WERNER 1840/41:270) in einer untergründig erotisch-koitalen Metaphorik beschrieben. Werners Überhöhung der WandaFigur zur zögerlich gewalttätigen Erlöserin des defizitären Mannes wird unter anderem Vorzeichen erst sechzig Jahre später in Sacher-Masochs Venus im Pelz fortgeführt. Dagegen greift der 1831 im Zusammenhang der deutschen Poleneuphorie erscheinende Roman Wanda Wielopolska oder das Recht der Gewaltigen von Wilhelm von Chézy wieder auf die nationale Dimension der Figur zurück. Der Roman stellt diese in ein dreifaches historisches Verweissystem: Während die Wanda-Figur auf die mittelalterliche Legende verweist, spielt die Handlung des Romans unmittelbar vor der ersten Teilung Polens im Jahr 1772; durch die Entstehungszeit bildet der polnische Aufstand von 1830 das aktuelle Referenzthema. Als verwitwete Gräfin eines der angesehensten 
polnischen Adelsgeschlechter wird die nicht mehr junge Wanda des Romans zur ,matka polka', zur mütterlichen Galionsfigur der für Freiheit und Einheit kämpfenden Adelskonföderation. Allerdings dienen politische Vorgänge wie die Intervention Wandas am Hof Maria Theresias nur als Rahmen für melodramatische Verwicklungen und Verwechslungen, in deren Mitte eine ebenso besonnene wie kämpferische Wanda steht. Trotz einer ganzen Anzahl leidenschaftlicher Verehrer unterschiedlicher Nationalität zeichnet sie sich weniger durch ihre „herbstlichen Reize“ (CHÉZY 1831:32) als durch Weisheit, Patriotismus und Beharrlichkeit aus. Nur als sie auf den Geliebten von einst, den mittlerweile mordenden und vergewaltigenden Obristen Augustin von Lazárfeld, stößt, lässt sie sich, obgleich sie seine Verbrechen kennt, von ihren Gefühlen überwältigen: ,,und die Heldin ward in der Aufregung der Seele und des Herzens zum Weibe“ (CHÉZY 1831:48). Zerrissen von Abscheu und Eifersucht, gewinnt sie schnell ihre unweibliche Selbstkontrolle zurück und verlangt dem Geliebten das Versprechen ab, sie erst dann wiederzusehen, wenn er durch Unterstützung der polnischen Freiheit seine Schuld gesühnt hat. Die Läuterung gelingt, doch fällt der wieder in Ehren stehende Obrist kurz vor seiner Eheschließung mit Wanda, die statt mit „weibischer Angst“ mit „männlicher Thätigkeit" (CHÉZY 1831:237) um Krakau kämpft, dem Anschlag eines einst von ihm gedemütigten Gefolgsmannes zum Opfer. Chézys dem bürgerlichen Frauenbild entgegenstehende , männliche ' Wanda markiert eine interessante Zwischenstufe in der Transformation der Figurenkonzeption von sittlicher zu erotischer Überlegenheit: Ähnlich wie bei der Läuterung des Obristen macht sie nämlich mehrfach die ihr durchaus zur Verfügung stehende kokettierende Attitüde zu einem Instrument des Freiheitskampfes: , ,[...] wusste sie doch [...] die Wirkungen dieser Koketterie hervorzuzaubern“ (CHÉZY 1831:231). Die vaterländischen Verdienste sind dann auch der Grund, weshalb ihr seitens der Kirche ihre Affären und Verführungen vergeben werden. In ausdrücklicher Absetzung von einem Liebestod, wie er etwa bei Werner dargestellt wird, lässt Chézy die nach der politischen und militärischen Niederlage zur Selbsttötung entschlossene Wanda betonen, dass sie nicht am Grab des Geliebten und mit „lüsternen Blicken in die rollenden Wogen der Weichsel“, sondern „mit Römermuth am Grabe der Freiheit" (CHÉZY 1831:287) sterbe. Das metaphysische Liebesglück wird hier durch die Hoffnung auf eine spätere Befreiung Polens ersetzt.

Das spezifische Geschlechterverhältnis, das Leopold von Sacher-Masochs Novelle Venus im Pelz 1870 zur Urszene des Masochismus machte, lässt sich nicht allein aus der Tradition der Wanda-Figur herleiten: Die dämonisch-verführerischen Frauengestalten der Romantik und die masochistischen Konstel- 
lationen im Werk Iwan Turgenjews (POLUBOJARINOVA 2002:236) haben deutliche Spuren hinterlassen, zudem hat die sexuelle Konstitution des Autors entscheidenden Anteil an der Figurenkonzeption. Dennoch ist es kein Zufall, dass die zur peitschenden Despotin gemachte junge und schöne Witwe aus Lemberg den Namen Wanda von Dunajew trägt. ${ }^{3}$ Auch die Ausstaffierung des zu ihrem Sklaven Gregor werdenden schwärmerischen Kunstdilettanten Severin von Kusiewski mit einem „Krakauerkostüm“ (SACHER-MASOCH 1987:99) sowie der Ort ihres Zusammentreffens, ein Kurbad in den Karpaten, weisen auf das Umfeld der Wanda-Legende. Der aus Galizien stammende Sacher-Masoch könnte die Legende in ihrer traditionellen Form oder durch Werners Drama kennengelernt haben. Die rückblickend in Form von Tagebuchpassagen beschriebene Beziehung zwischen beiden Figuren geht von Severins sinnlichen Projektionen auf eine Venusstatue aus, die er, von der frivolen Kostümierung seiner Nachbarin Wanda angeregt, auf diese überträgt. Wanda erweist sich auch weiterhin als herausfordernde Mitspielerin. Beeindruckt vom Ernst, mit dem Severin seinen erotischen Passionen folgt, nimmt sie nach einigem Zögern die ihr zugewiesene Rolle einer in Pelz gehüllten Herrin an und beginnt, an den ihr abverlangten Grausamkeiten Vergnügen zu empfinden. Die Novelle entwirft ein dualistisches Weltbild, in dem sich Männliches und Weibliches als christlich sublimiertes Begehren des Aufschubs und griechisch-antiker Hedonismus unvereinbar gegenüberstehen. Dieses Geschlechterverhältnis lässt Severin zufolge einem Mann nur die Wahl, ,der Tyrann oder der Sklave des Weibes“ (SACHER-MASOCH 1987:15) zu sein. Als prototypisches Weib ausgewiesen, folgt Wanda ihren Affekten, plädiert für Genuss ohne Schmerz und bekennt sich zu „kalte[r] Koketterie“ (SACHER-MASOCH 1987:21) und „Charakterlosigkeit“ (SACHER-MASOCH 1987:72). Um die launige Geliebte dauerhaft an sich zu binden, unterwirft sich Severin, geleitet von weiblichen Gewalterfahrungen seiner Kindheit, als rechtloser Lakai, der unter vertraglich vereinbarten Peitschenhieben höchste Befriedigung findet. Das unmännliche Gebaren ihres Verehrers lässt Wandas Sympathien allerdings schnell abkühlen. Dagegen gibt der „Ritter vom Geiste“ (SACHER-MASOCH 1987:27) erst dann seine Entbehrungsphantasien auf und findet zu einer ,normalen', d.h. autoritären Männlichkeit, als die mit ihm aus dem slawischen in den antiken Kultur-

3 Die Nationalität dieser Wanda-Figur bleibt unbestimmt slawisch. Der Nachname klingt russisch (POLUBOJARINOVA 2002:226), etymologisch verweist er auf die Donau (in verschiedenen slawischen Sprachen: Dunaj), vielleicht auch auf den Fluss Dunajec, der, aus den Karpaten kommend, unweit Krakaus Galizien durchfließt und in die Weichsel mündet. 
kreis reisende Wanda das Auspeitschen einem ihrer dominant-männlichen Liebhaber überlässt. Die Überlegenheit Wandas in Venus im Pelz ist nicht genuin, sondern folgt einer männlichen Regie und wird am Ende der Novelle als Produkt einer krankhaften männlichen Vorstellung desavouiert. Die verborgene Dominanz des sich scheinbar selbst erniedrigenden Mannes beweist sich auch darin, dass Severins Begehren nicht Wanda gilt, sondern diese lediglich als fetischtragendes lebendes Surrogat von Kunstwerken fungiert. Solange sie sich seiner Inszenierung fügt, bleibt sie Objekt seiner Projektion. Erst als Wanda sich dieser entzieht, erlangt sie eine autonome Position. Dies erlebt der entsetzte Severin als Sadismus, den er rückblickend jedoch als heilsame Lösung aus der Welt der Ideen und als Initiation in die väterliche Welt der bürgerlichen Pflicht interpretiert. So gesehen trägt die Novelle Züge eines Bildungsromans. Auf der Gegenwartsebene der Novelle ist das Geschlechterverhältnis genau umgekehrt: Entsprechend seines Grundsatzes, eine Frau könne niemals Gefährtin des Mannes sein, tritt nun Severin als peitschender Ehemann einer hingebungsvollen Gattin auf.

An Sacher-Masoch anschließende Figurenkonzeptionen gibt es in der Literatur vor und nach 1900 unzählige Male, jedoch gewöhnlich ohne Verwendung einer Wanda-Figur. So etwa in Franz Kafkas Erzählung Die Verwandlung, in welcher der als Ungeziefer erwachende Gregor Samsa ein erhöht hängendes Porträt einer Dame mit Pelzfetisch betrachtet. ${ }^{4}$ Deutlich an die Wanda-Tradition knüpft jedoch C. JÜRGENS' 1902 erschienener erotischer Roman Wanda. Domina mea severa an. Die Handlung ist an die mecklenburgische Ostseeküste verlegt. Jürgens' trivialisierte Neufassung von Sacher-Masochs Venus im Pelz übernimmt neben der pelztragenden Polin zahlreiche weitere Motive, bis hin zum bekenntnishaften Tagebuchstil des Ich-Erzählers. Ohne lange Vorrede kommt der Landadlige Johannes von Ketzin auf seine quälende Leidenschaft zu sprechen:„Könnte ich dein Sklave sein, und du meine Gebieterin, grausam, streng und unerbittlich!"5 Bei der Angeflehten handelt es sich um Wanda von Krszeminska, eine rothaarige Exilpolin mit reizenden Stiefelchen, viel Pelz und exotisch-zügellosem Lebenswandel. Sie wird von Ketzin beim Wildern in seinen Wäldern gestellt und peitscht den augenblicklich in sie Verliebten bereits während der ersten Begegnung. Andeutungen einer antirussischen Verschwörung einiger dubioser Polen dienen außer als dramatisches Requisit der

$4 \quad$ Die Figur des Gregor Samsa bezieht sich direkt auf Sa(cher)-Mas(ochs) Figur des Severin alias Gregor (RUDLOFF 1997).

5 JÜRGENS (1902:4); im Original alles hervorgehoben. 
Vorbereitung des Romanschlusses, den Jürgens nicht aus der Dynamik der Liebesbeziehung zwischen Ketzin und Wanda entwickelt, sondern von außen herbeiführt: Verraten von einem eifersüchtigen Polen, wird Wanda verhaftet, beim anschließenden Schusswechsel stirbt sie an Ketzins Seite. Der gravierendste Unterschied zu Sacher-Masochs Rollenspielen ist jedoch die Umkehrung der Emotionskurve der weiblichen Figur. Wandas Brutalität entspringt zunächst keiner männlichen Inszenierung, sondern liegt der schönen Polin ebenso in der Natur wie dem zartsinnigen Deutschen die masochistische Unterwerfung. Diese bedarf auch keiner Heilung, sondern lässt sich letztendlich sogar mit Wandas aufkommender Gegenliebe harmonisieren. Während Sacher-Masoch seinen Protagonisten in die patriarchalische Ordnung zurückbiegt, lässt Jürgens ihn alle Offerten zu einem bürgerlichen Familienglück brüsk ausschlagen. Dafür führt er als genaues Gegenbild Wandas die Figur der Martha ein, eine Ketzin seit langem treu und tugendhaft ergebene Gefährtin, deren Position zwischen Partnerin und Bediensteter nicht sicher auszumachen ist. Martha verkörpert das Ideal der bürgerlichen Frau: Schamhaft, schlicht und pflichtbewusst nimmt sie Ketzins Affäre mit der Polin schweigend hin. Doch gerade wegen ihrer selbstlosen Liebe hat sie, obgleich von Ketzin ein Kind erwartend, gegenüber der neben Männern auch Löwen züchtigenden Polin keine Chance. Wandas Verhältnis zu Ketzin ist widersprüchlich dargestellt. Zunächst ist sie eine rücksichtslose Despotin, die ihn mit einem anderen Liebhaber quält. Selbst scheint sie auf ein autoritäres Männerbild fixiert zu sein, das sie jedoch ihren langsam erwachenden Gefühlen zu dem beharrlichen Stiefelfetischisten unterordnet. Diese ihn allzu sehr an Martha erinnernde Neigung Wandas irritiert Ketzin in seinem masochistischen Begehren nach Demütigung. Aus diesem Grund nehmen, in Umkehrung zu Venus im Pelz, die Auspeitschungen erst gegen Ende der Handlung den Charakter einer Inszenierung für den geliebten Sklaven an. Bei Jürgens ist lediglich angedeutet, was bei Sacher-Masoch offensichtlich war: Aus Neigung unterwirft sich die Frau den Bedingungen des männlichen Begehrens. Dies galt, allerdings aus ökonomischen Ursachen, auch für die scheinbare Überlegenheit der Wanda-Figur in Fontanes Stine.

\section{Transformation der Figur im diskursiven Kontext}

Die chronologischen Durchgänge durch verschiedene Darstellungsvarianten von Weiblichkeit, die an die deutsche Wanda-Figur geknüpft wurden, haben eine zunehmende Dominanz und unterschwellige oder robuste Überlegenheit der männlichen Figur aufgezeigt. Wird das Begehren des Liebhabers in dem 
anonymen Roman, bei Werner, Schiller, Heyse und Chézy wegen der Bindungen Wandas an ihr Vaterland abgewiesen oder aufgeschoben, setzt es sich in den späteren Texten, angefangen von May über Sacher-Masoch, Fontane bis Jürgens im Gestus vermeintlicher (sozialer oder sexueller) Unterlegenheit durch. Dieser Registerwechsel von der heroischen zur erotischen Figur vollzieht sich in der Mitte des Jahrhunderts; mögliche Erklärungen dafür sind der sich verändernde gesellschaftlich-politische Pol(inn)endiskurs in Deutschland und die zunehmende Faszinationskraft außer- oder gegenbürgerlicher Weiblichkeit. Chézys Roman und Mays Novelle können als Übergangsformen innerhalb dieses Registerwechsels angesehen werden. In der heroischen Konzeption der Wanda-Figur lässt sich mit KolLMANNs Kategorie des „heroischen Geschlechts“ (2004:221f.) eine Darstellungsform herausheben, in der die Heroin in einem (vergeblichen) „Kampf um Subjektautonomie“ eine „Lösbarkeitsillusion“ in Gestalt eines ,dritten Ort[es], an dem ihre Liebe zu verwirklichen sei“", sucht. Dies gilt von den untersuchten Texten vor allem für Werners Wanda-Tragödie, während der anonyme Roman von 1804 (welcher der Figur lediglich vor ihrer Begegnung mit dem späteren Geliebten ein erstaunliches $\mathrm{Ma}$ an Autonomie zubilligt), Schillers und Heyses Balladen und Arnims Persiflage dem konventionellen Modell des Heroischen verpflichtet sind und Wanda der vaterländischen und väterlichen Ordnung opfern. ${ }^{6}$ Bei Chézy ergänzen sich, da der Geliebte dem eigenen Volk angehört, patriotische Pflicht und individuelle Neigung. Über den Registerwechsel hinweg weisen Werners und Sacher-Masochs Wanda-Figur Parallelen auf. Sacher-Masochs Wanda unterwirft sich zunächst der ihr zugewiesenen Überlegenheitsrolle, tritt jedoch mehr und mehr im Rekurs auf ihr von der männlichen Rollenvorgabe abweichendes Weiblichkeitsverständnis aus dieser heraus. In dieser Selbstständigkeit ist sie Werners Wanda-Figur vergleichbar, die sich nicht nur gegen eine, sondern gegen zwei männlich repräsentierte Anforderungen (Vaterland und Werbung) zu behaupten hat. Beide Texte stellen die Gewalterfahrung, die der weibliche Autonomiegewinn für den männlichen Liebhaber mit sich bringt, als schmerzhafte Initiation des Mannes in eine jenseitig-metaphysische (Werner) oder diesseitig-patriarchalische Ordnung (Sacher-Masoch) dar. Eine vergleichbare latente weibliche Autonomie und gewaltsame männliche Initiation ist in

6 „Die Opferstruktur kann verschiedene Formen annehmen, insbesondere sind es Frauen, die vor dem Hintergrund des symbolischen Gewichts eines (väterlichen, familiären, sozialen, göttlichen) Gesetzes als ,geopferte Stütze ' in einem solchen Sinnbildungsprozeß fungieren." (HoFf 1988:255) Dies lässt sich, wie Hoff zeigt, aber nicht durch eine männliche Autorschaft erklären. 
Mays, Fontanes und Jürgens' Wanda-Erzählungen nicht zu finden, die eher eine Lösung im Modus weiblichen Sich-Arrangierens verfolgen. Dass es sich bei der erotischen Wanda-Figur nicht um eine völlig andere, sondern um eine Transformation der heroischen Figur handelt, zeigen nicht nur die figurenkonstitutiven Überlegenheitsmerkmale Gewalt, Affektkontrolle und Anziehung, sondern auch die späten, ironisch gebrochenen Referenzen der erotischen Konzeption auf die heroische Tradition in Fontanes Stine.

Mit der um 1800 einsetzenden Transformation der Sexualitäts- und Eheauffassungen von einer öffentlich-familialen, weitgehend durch Macht- und Eigentumsfragen bestimmten Angelegenheit zu einer individuellen Angelegenheit privater Leidenschaft (FOUCAULT 1977:170-183) verschiebt sich die Überlegenheit der deutschen Wanda-Figur mehr und mehr von einem dynastischen, also kollektiven, zu einem privaten und zwischengeschlechtlichen Konfliktfeld. Damit verbunden ist die für die deutsche Wanda-Figur um 1800 (freilich nicht nur für diese) charakteristische Konfliktkonstellation einer beidseitigen, die kollektiv-nationale Konfrontation konterkarierende Liebe zwischen dem deutschen Ritter und der polnischen Fürstin. Das - im Unterschied zur polnischen Wanda-Figur - für die deutsche Wanda charakteristische Motiv wechselseitiger Liebe tritt bereits am Beginn des 18. Jahrhunderts auf (DEGEN 2007) und setzt sich nach 1800 als bestimmende Konstellation durch. Es geht nun nicht mehr um eine dynastische Allianz, die Wanda ausschlägt, sondern um eine leidenschaftliche Liebe zwischen Individuen verfeindeter Völker. Ähnlich wie in Kleists Penthesileia oder Schillers Jungfrau von Orleans konzentriert sich dabei die Darstellung auf das Abwägen der Heldin zwischen persönlicher Neigung und sozialer Pflicht. Die in der traditionellen Wanda-Legende auf den äußeren Feind gerichtete Gewalt wird in der heroischen Konzeption mehr und mehr nach innen, gegen die eigenen Empfindungen gerichtet. Die vordem im Kampf bewiesene kollektive Überlegenheit des polnischen Heeres wird in Selbstdisziplinierung und Affektkontrolle transformiert. Diese Rückbiegung der ursprünglich auf den männlichen Angreifer gerichteten Gewalt der Frau auf sich selbst findet eine Fortführung in der erzwungenen Rollengewalt der Sacher-Masoch'schen Domina. Neben den epochalen Verschiebungen innerhalb der Gefühls- und Liebesdiskurse um 1800 lässt sich das allmähliche Ausblenden der kollektiv-nationalen Dimension des Konflikts in den deutschsprachigen Wanda-Texten auch aus der politischen Gesamtsituation erklären. Nach den drei Teilungen Polens hätte die (deutsche) Darstellung eines polnischen Sieges über ein deutsches Heer vermutlich anachronistisch gewirkt. Zudem verlief der Prozess der deutschen Nationwerdung nach einer kurzen Welle der Poleneuphorie seit Mitte des 19. Jahrhunderts in massiver 
Abwehr nationaler Bestrebungen auf polnischer Seite (KоCH 2002:30f.). Ein Wanda-Text mit deutscher Niederlage hätte da kaum auf Zuspruch hoffen dürfen. Der deutsche Polendiskurs verzeichnete bekanntermaßen im Verlauf des 19. Jahrhunderts insgesamt eine deutliche Abwertung des Nachbarvolkes. Dies betraf auch das eng mit der Wanda-Figur verbundene Motiv der ,schönen Polin' ${ }^{7}$ Während in der polnischen Literatur der Wanda-Legende eine zentrale nationale Identitäts- und Legitimationsfunktion zukam, fiel sie als moralisches Identifikationsangebot in der deutschen Literatur nach 1830 fast ganz aus. Stattdessen wurde die von einer ethischen Vorbildfunktion gelöste Figur der Polin Wanda zu einer erotischen Projektionsfläche (männlicher) außerbürgerlicher Weiblichkeitsvorstellungen. Für sie dürfte gegolten haben, was für das im 19. Jahrhundert nicht seltene Motiv der polnischen Frau am Ende des Jahrhunderts gegolten hat: die charakteristische Verbindung von „Dominanz und einer relativ freizügigen Sexualität“ mit „,geradezu heroische[n] kriegerische[n] Aktivitäten“. ${ }^{8}$ Wie an die am Anfang erwähnte Angel-Imago der Wanda-Tradition anschließend, heißt es beispielsweise 1890 über die Polin als solche: „Durch sirenenhafte Liebenswürdigkeit alle Herzen bezwingend, weiß sie gelegentlich durch männliches, ja amazonenhaftes Auftreten zu imponieren, durch ungezügelte Leidenschaftlichkeit und Härte sich gefürchtet zu machen [...].“ (HellWALD 1890:219, zit. nach KoCH 2002:219) Die genannten Wirkungen der bürgerlich-empfindsamen Gefühlskultur um 1800 und die Veränderungen innerhalb des deutschen Polendiskurses führten während des 19. Jahrhunderts $\mathrm{zu}$ den oben beschriebenen Veränderungen der deutschen Wanda-Figur. Der kollektiv-dynastische Konflikt wurde zunehmend durch eine als individuell dargestellte Liebestragödie überlagert. Dabei wurde Wanda tendenziell von ihrem Herkunftskollektiv abgesetzt und dem Geliebten angenähert. Drittens löste sich, ablesbar auch an der allmählichen Verbreitung des Vornamens Wanda im deutschen Sprachraum, die Figur von der historischen

7 Zum Motiv der ,schönen Polin', das in den wenigen Untersuchungen ohne Verweis auf die Wanda-Figur behandelt wird, vgl. SZYROCKI (1975:5) und ORŁOWSKI (1996:191-231), die zahlreiche Belege für die Sexualisierung und latente Dämonisierung anführen; für dieses Motiv scheint eine besondere erotische Konnotation schon vor 1800 üblich gewesen zu sein. - Der Umschwung des deutschen Polendiskurses in der zweiten Hälfte des 19. Jahrhunderts lässt sich für die Figur der Wanda besonders gut dann zeigen, wenn ein Autor mehrfach in seinem Werk auf die Figur zurückgreift (DEGEN 2007).

8 So Косн (2002:188) für die Zeitschrift Die Gartenlaube. 
Andreas Degen

Legende. ${ }^{9}$ Damit verbunden war die Aufgabe Krakaus bzw. Polens als Handlungsort: Alle nach 1851 entstehenden deutschsprachigen Wanda-Texte spielen außerhalb Polens. ${ }^{10}$ Viertens wurde die polnische Provenienz Wandas zu einem exotischen Accessoire herabgestuft, das - fünftens - dazu diente, sie als Gegenfigur zur bürgerlichen Ordnung und zum bürgerlichen Frauenbild zu etablieren. Damit vollzog sich in der zweiten Hälfte des 19. Jahrhunderts eine Transformation der Figur von einer sittlichen zu einer erotischen Überlegenheit, wobei die Merkmale Abwehr und Gewalt in modifizierter Form erhalten blieben. Innerhalb dieser Transformation wurde jedoch die Überlegenheit der weiblichen Figur immer stärker unterhöhlt: nicht national, sondern geschlechtsspezifisch. Die Wanda-Figur verlor im letzten Drittel des 19. Jahrhunderts ihre Überlegenheit und nahm die Position einer mehr oder weniger passiven Erfüllungsgehilfin männlichen Begehrens ein. Ihr erotisch getrimmter Gestus der Überlegenheit folgte in Wahrheit den Phantasien und Instruktionen ihrer männlichen Partner.

\section{Literatur}

[Anonymus] (1705): Venda, Reine de Pologne. Histoire Galante. Den Haag.

[ANONYMus] (1804): Wanda, Fürstin von Krakau, oder die Opfer der Liebe. Nach der wahren Geschichte romantisch bearbeitet. Wien.

BRUnS, KARIN / PARR, Rolf / WÜLFING, Wulf (eds.) (1991): Historische Mythologie der Deutschen. München.

ChÉZy, Wilhelm von (1831): Wanda Wielopolska oder Das Recht der Gewaltigen. Erzählung aus den Zeiten der ersten Theilung Polens. Stuttgart.

Chochanovius, IoAnnis (1987): Eligarium Liber I, Elegia XV. In: Fieguth, Rolf (ed.): Jan Kochanowski. Ioannis Chochanovius (1530-1584). Materialien des Freiburger Symposiums 1984. Fribourg, 158-163.

Degen, ANDREAS (2007): Der Sieg der Krakauer Fürstin Wanda über den deutschen Ritter Rüdiger. Zur deutschen Rezeption eines antideutschen Mythos vom 18. Jahrhundert bis zur Gegenwart. In: Albrecht, Dietmar / Degen, Andreas / Neumann, Bernd / TAlarczyK, Andrzej (eds.): Provinz als Zentrum. Aachen [erscheint demnächst].

9 SEIBICKEs (2003:411) frühester Beleg für die Verwendung des Vornamens Wanda im deutschen Sprachraum datiert, abgesehen von Zacharias Werners Drama Wan$d a$, von 1819; für die zweite Hälfte des 19. Jahrhunderts häufen sich die Belege.

10 Eine Ausnahme ist Leopold von Sacher-Masochs Novelle Venus im Pelz, deren erste Hälfte im österreichischen Teil Polens spielt. 
Patria und Peitsche. Weiblichkeitsentwürfe in der deutschen Wanda-Figur

Fontane, THEODOR (1964): Sämtliche Werke. Romane, Erzählungen, Gedichte. Bd. 6. München.

- (2000): Stine. Hrsg. von Christine Hehle. Berlin.

Foucault, Michel (1977): Der Wille zum Wissen. Sexualität und Wahrheit. Bd. 1. Frankfurt (M.).

FREVERT, Ute (1995): „Mann und Weib, und Weib und Mann“. Geschlechter-Differenzen in der Moderne. München.

Hauff, Wilhelm (1970): Die letzten Ritter von Marienburg. In: Hauff, Wilhelm: Sämtliche Werke. Bd. 2: Märchen, Novellen. München, 584-639.

Hellwald, F. von (1890): Die Welt der Slawen. Berlin.

Heyse, Paul (1980): Werke. Mit einem Essay von Theodor Fontane. Hrsg. von Bernhard und Johanna Knick und Hildegard Korth. Bd. I: Gedichte. Frankfurt (M.).

Hoff, Dagmar von (1988): Die Inszenierung des ,Frauenopfers ' in Dramen von Autorinnen um 1800. In: Pelz, ANNEGREt / SCHUller, MarianNe / STEPHAN, INGE et alii (eds.): Frauen, Literatur, Politik. Hamburg, 255-262.

JürgENS, C. (1902): Wanda. Domina mea severa. Dresden.

Klin, Eugeniusz (1996): Das Drama „Wanda, Fürstin von Polen oder unglückliche Heirat" von G. F. Wurrwitz. In: KUNICKI, WOJCIECH (ed.): Aufklärung in Schlesien im europäischen Spannungsfeld. Traditionen - Diskurse - Wirkungen. Wrocław, 243-247.

Koch, Angela (2002): DruckBilder. Stereotype und Geschlechtercodes in den antipolnischen Diskursen der „Gartenlaube“ (1870-1930). Köln/Weimar/Wien.

Kollmann, ANETt (2004): Gepanzerte Empfindsamkeit. Helden in Frauengestalten um 1800. Heidelberg.

KOZIEŁEK, GERARD (1967): Das dramatische Werk Zacharias Werners. Wrocław (=Prace Wroctawskiego Towarzystwa Naukowego. Seria A 120).

- (1976): Ludwig Gotthard Theobul Kosegartens Romanze von der schönen Polenkönigin. In: Germanica Wratislaviensia 26:29-43.

- (1989): Das Polenbild der Deutschen. 1772-1848. In: Das Polenbild der Deutschen. 1772-1848. Anthologie. Hrsg. von Gerard Koziełek mit einem Geleitwort von Wolfgang Drost. Heidelberg, 11-70.

LANGe, Sigrid (1992): Nachwort. In: LANGe, SigRID (ed.): Ob die Weiber Menschen sind. Geschlechterdebatten um 1800. Leipzig, 411-432.

Lauterbach, SAmuel Friedrich (1993): Pohlnische Chronicke [...], Kapitel 4. In: Schillers Werke. Nationalausgabe. Bd. 2 II B: Gedichte. Anmerkungen zu Bd. 2 I. Hrsg. von Georg Kurscheidt und Norbert Oellers. Weimar, 263f.

LiedKe, HeRBERT R. (1965): Vorstudien Achim von Arnims zur „Gräfin Dolores“, II. Teil. Entwurf und Quellen zur „Geschichte der verlorenen Erbprinzessin Wenda“. In: Jahrbuch des Freien Deutschen Hochstifts:237-313.

MAY, KARL (1901): Wanda. Novelle. Dresden. 
Mortkowiczówna, Hanna (1927): Podanie o Wandzie. Dzieje watku literackiego (=Studia z Zakresu Historii Literatury Polskiej 7). Warszawa.

OrŁowski, Hubert (1996): „Polnische Wirt(h)schaft“. Zum deutschen Polendiskurs der Neuzeit. Wiesbaden.

POlubOJARINOVA, LARISSA (2002): Sacher-Masoch und die Slawen. In: SPÖRK, INGRID / STROHMAIER, AlEXANDRA (eds.): Leopold von Sacher-Masoch. Graz/Wien, 222-250.

RudLOFF, Holger (1997): Gregor Samsa und seine Brüder. Kafka - Sacher-Masoch Thomas Mann. Würzburg.

SACHer-MASOCh, LeOpold von (1987): Venus im Pelz. Die Liebe des Plato. München.

SCHABERT, INA (1995): Gender als Kategorie einer neuen Literaturgeschichtsschreibung. In: BusSMANN, HADUMOD / HoFF, RENATE (eds.): Genus. Zur Geschlechterdifferenz in den Kulturwissenschaften. Stuttgart, 162-205.

SCHILlER, FrIEDRICH (1993): In: Schillers Werke. Nationalausgabe. Bd. 2 I: Gedichte. Hrsg. von Georg Kurscheidt und Norbert Oellers. Weimar.

SEIBICKE, WiLfRIED (2003): Historisches deutsches Vornamenbuch. Bd. IV. Berlin/New York.

SzYrocki, Marian (1975): Das Bild des Polen in der deutschen Literatur und das Bild des Deutschen in der Literatur der VR Polen. Düsseldorf.

WERNER, ZACHARIAS (1840/41): Wanda, Königin der Sarmaten. Eine romantische Tragödie mit Gesang in fünf Akten. In: WERNER, ZACHARIAS: Sämtliche Werke, aus seinem handschriftlichen Nachlaß hrsg. von seinen Freunden. Bd. VI. Grimma, 187-271. 